\title{
Impella-Induced Incessant Ventricular Tachycardia
}

\author{
Amir Kaki, MD, ${ }^{1}$ Ahmed Subahi, MD, ${ }^{2}$ Mohamed Shokr, MD, ${ }^{3}$ Walid Ibrahim, MD, ${ }^{2}$ Ahmed Yassin, MD, ${ }^{2}$ \\ Reema Hasan, MD, ${ }^{4}$ M. Chadi Alraies, MD, ${ }^{1}$ Theodore Schreiber, MD ${ }^{1}$ \\ ${ }^{1}$ Department of Interventional Cardiology, Wayne State University School of Medicine, Detroit Medical Center Heart Hospital, Detroit, MI \\ ${ }^{2}$ Department of Internal Medicine, Wayne State University/Detroit Medical Center, Detroit, MI ${ }^{3}$ Department of Cardiology, Wayne State \\ University/Detroit Medical Center, Detroit, MI ${ }^{4}$ Department of Cardiovascular Medicine, University of Michigan, Ann Arbor, MI
}

Background: The Impella 2.5 and Impella Cardiac Power (CP) devices (ABIOMED) are used to provide mechanical circulatory support for high-risk percutaneous coronary interventions or cardiogenic shock as a bridge to recovery or destination therapy. The Impella device has shown both efficacy and safety in patients presenting with cardiogenic shock. Performing clinical and hemodynamic assessments of patients presenting with cardiogenic shock is an important step to determine if mechanical circulatory support with an Impella device is indicated.

Case Report: A 62-year-old male presented with cardiogenic shock requiring Impella device support. Two days later, the patient developed incessant ventricular tachycardia. Transthoracic echocardiography showed that the Impella device had migrated out of the ventricular cavity. Once the position of the Impella device was adjusted, ventricular tachycardia immediately resolved.

Conclusion: Our case emphasizes the importance of using imaging modalities such as transthoracic echocardiography to ensure correct positioning of an Impella device in the left ventricle to avoid complications.

Keywords: Arrhythmias, assisted circulation, heart-assist devices, shock-cardiogenic, tachycardia-ventricular

Address correspondence to M. Chadi Alraies, MD, Department of Interventional Cardiology, Wayne State University School of Medicine, Detroit Medical Center, Detroit Medical Center Heart Hospital, 311 Mack Ave., Detroit, MI 48201. Tel: (216) 255-0008. Email: alraies@hotmail.com

\section{INTRODUCTION}

Use of the Impella 2.5 and Impella Cardiac Power (CP) devices (ABIOMED) to provide mechanical circulatory support for patients in cardiogenic shock and patients undergoing high-risk percutaneous coronary interventions in the United States has increased since US Food and Drug Administration (FDA) approval in 2015. ${ }^{1-3}$ Impella device placement prior to high-risk percutaneous coronary interventions provides hemodynamic support and myocardial ischemic protection, with a resultant considerable survival benefit. ${ }^{1-3}$ Manufacturer guidelines indicate that optimal placement for the Impella inlet is $35 \mathrm{~mm}$ below the aortic valve annulus away from papillary muscle and the subannular structures. ${ }^{1,2}$ The Impella outlet area should be well above the aortic valve. ${ }^{1,2}$ Initial Impella device placement is usually performed under fluoroscopy guidance in the catherization laboratory. Although interventional cardiologists are gaining more experience with the device, complications such as high purge pressures, sensor failure, suction episodes, low volume, device thrombosis, and aortic or mitral valve injury are being reported in the literature. ${ }^{1,2}$ Other access-related complications include bleeding and vascular complications (limb ischemia, pseudoaneurysm, and arteriovenous fistula). ${ }^{1,2,4-6}$ However, as with any percutaneous mechanical circulatory support device, Impella displacement is the most common complication and often occurs secondary to patient movement or transportation. ${ }^{1,2}$ The device can migrate deep into the ventricular cavity or out into the aortic root, placing both the inlet and the outlet inside or outside the ventricular cavity, respectively, with subsequent failure of ventricular unloading. Device malpositioning can result in direct contact with and hence mechanical irritation of adjacent cardiac structures including the myocardial wall, papillary muscles, and mitral or aortic valve cusps.

We present a case of incessant ventricular tachycardia resulting from improper Impella device positioning that immediately resolved after repositioning under echocardiographic guidance.

\section{CASE REPORT}

A 62-year-old male with no significant medical history presented to the emergency department (ED) with a 3day history of angina on exertion associated with acute onset progressive dyspnea on exertion. Laboratory workup revealed normal complete blood count, bleeding profile, basic metabolic panel, and thyroid-stimulating hormone. He had elevated troponin I of $3 \mathrm{ng} / \mathrm{mL}$ (normal, $<0.057 \mathrm{ng} / \mathrm{mL}$ ). Electrocardiogram (ECG) showed diffuse $T$ wave inversion and ST segment depression. While the patient was in the $\mathrm{ED}$, he became hypotensive with blood pressure of $80 / 60$ $\mathrm{mmHg}$, heart rate of $120 \mathrm{bpm}$, and oxygen saturation of $85 \%$ on $6 \mathrm{~L}$ nasal cannula. He was urgently transferred to the catheterization laboratory for emergent left heart catheterization and cardiogenic shock management. Coronary 


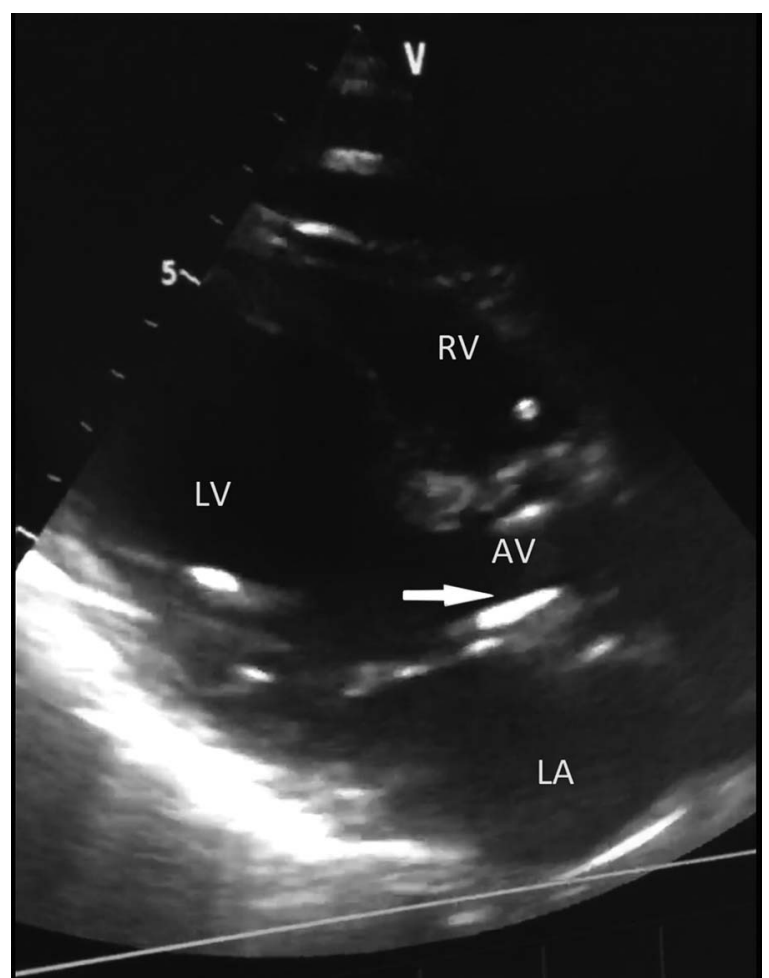

Figure 1. Transthoracic echocardiogram parasternal long axis view showing the Impella catheter tip above the aortic valve (arrow). AV, aortic valve; LA, left atrium; $\mathrm{LV}$, left ventricle; $\mathrm{RV}$, right ventricle.

angiogram showed heavily calcified left main coronary artery with $99 \%$ stenosis in the distal segment. The left circumflex artery had high-grade stenosis in the ostial segment. The left anterior descending artery also had stenosis in the ostial segment of $90 \%$ and in the mid segment of $50 \%$. Left to right collaterals suggested chronic total occlusion of the right coronary artery. Left ventricular ejection fraction was 10\%-15\%. Right heart catheterization hemodynamics suggested acute cardiogenic shock. Right atrial pressure was $24 / 21 \mathrm{mmHg}$, right ventricular pressure was $59 / 12 \mathrm{mmHg}$, pulmonary artery pressure was $52 / 30 \mathrm{mmHg}$, and cardiac output was $3.89 \mathrm{~L} / \mathrm{min}$. Given the patient's profound cardiogenic shock, mechanical circulatory support via Impella CP device was ordered. After device insertion, placement was confirmed by fluoroscopy. The patient was intubated and transferred to the cardiac care unit for observation pending multidisciplinary discussion between interventional cardiology and cardiothoracic surgery.

Two days later, the patient developed ventricular tachycardia arrest. Cardiopulmonary resuscitation and advanced cardiac life support (ACLS) were started and continued for 19 minutes with a total of 10 direct-current shocks. Intravenous amiodarone was administered per ACLS guidelines, followed by intravenous lidocaine, but ventricular tachycardia did not resolve. During ACLS, the cardiology team performed a bedside transthoracic echocardiogram (TTE) to check the Impella device position. TTE showed the Impella catheter inlet touching the aortic valve, and the catheter pigtail was close to the mitral valve (Figure 1). Under echocardiographic guidance, the position of the Impella device was adjusted so that the catheter inlet was advanced into the ventricular cavity and so that the outlet was supravalvular within the aortic root, leading to instantaneous resolution of the ventricular tachycardia. The patient did not receive any vasopressor support before or after Impella device placement. Laboratory workup showed normal electrolytes and renal function at the time of the cardiac arrest.

Given the patient's hemodynamics, presentation, arrhythmias, and severity of the coronary artery disease, his Society of Thoracic Surgeons risk score estimated a mortality rate of $13 \%$ and combined morbidity/mortality risk of $74 \%$. Based on these findings, the heart team was in favor of percutaneous coronary intervention vs bypass surgery. The patient underwent Impella-assisted high-risk percutaneous coronary intervention with stenting of his left main and left anterior descending arteries and balloon angioplasty of the ramus intermedius artery followed by Impella device removal 24 hours later. On day 7, repeat TTE showed an ejection fraction of $40 \%-45 \%$. The patient was discharged home on day 15 on aspirin, atorvastatin, clopidogrel, and carvedilol. Currently, he is under regular follow-up in the cardiology outpatient clinic.

\section{DISCUSSION}

The Impella 2.5 and Impella CP devices are approved by the US FDA for up to 6 days for patients in cardiogenic shock and up to 6 hours for high-risk coronary interventions. ${ }^{1,2}$ The Impella 2.5 and Impella CP devices provide direct cardiac unloading and antegrade flow up to 2.5 and $4.0 \mathrm{~L} / \mathrm{min}$, respectively. ${ }^{1,2}$ These two devices require single arterial access of $13 \mathrm{Fr}$ and $14 \mathrm{Fr}$, respectively. ${ }^{1,2}$ As noted earlier, the optimal device placement guidelines mandate that the Impella inlet should be $35 \mathrm{~mm}$ below the aortic valve annulus away from the subannular structures, and the Impella outlet should be well above the aortic valve (Figure 2). ${ }^{1,2}$ Potential complications resulting from improper positioning of an Impella device include hemolytic anemia requiring a blood transfusion, thrombocytopenia, and valvular injury. Improper positioning of the Impella device can also trigger arrhythmias because of mechanical irritation of the adjacent cardiac structures. ${ }^{1}$ These complications can be reduced with echocardiographic surveillance during and after Impella device placement. ${ }^{1,2}$

As the use of the mechanical circulatory support devices increases in patients with cardiogenic shock or patients undergoing high-risk percutaneous coronary interventions, cardiac arrest among this group of patients might pose a challenge to healthcare providers. In our patient, invasive blood pressure monitoring with the arterial line indicated inadequate forward Impella flow during the ventricular tachycardia arrest. Furthermore, Doppler flow assessment and point-of-care echocardiography confirmed the absence of meaningful forward flow. Therefore, external cardiac compression was initiated in compliance with the 2017 scientific statement from the American Heart Association. ${ }^{7}$

In our patient, several factors may have contributed to the development of the incessant ventricular tachycardia event. First, underlying coronary artery disease usually provides the appropriate substrates for the development of any cardiac arrhythmia. Also, the increase in the incidence of ventricular tachycardia early after insertion of ventricular support devices is well known and could be attributed to the fact that 


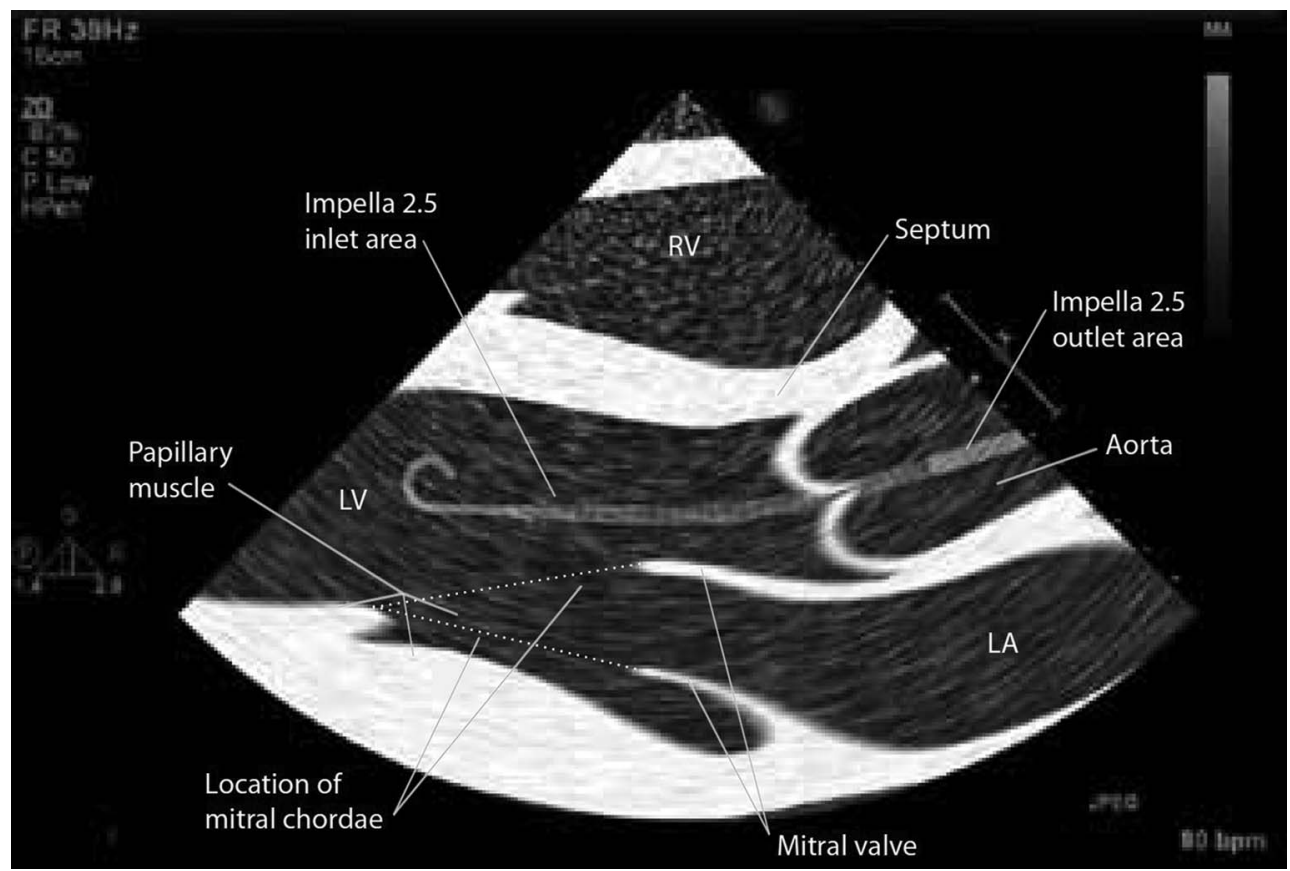

Figure 2. Transthoracic echocardiogram parasternal long axis view illustrating the correct positioning of an Impella device. LA, left atrium; LV, left ventricle; RV, right ventricle. (Image reproduced with the permission of ABIOMED.)

\begin{tabular}{|c|c|}
\hline Vent rate & 110 \\
\hline $\mathrm{PR}$ interval & 122 \\
\hline QRS duration & 8 \\
\hline QT/QTc & $352 / 476$ \\
\hline P-R-T axes & $48 \quad 74$ \\
\hline
\end{tabular}

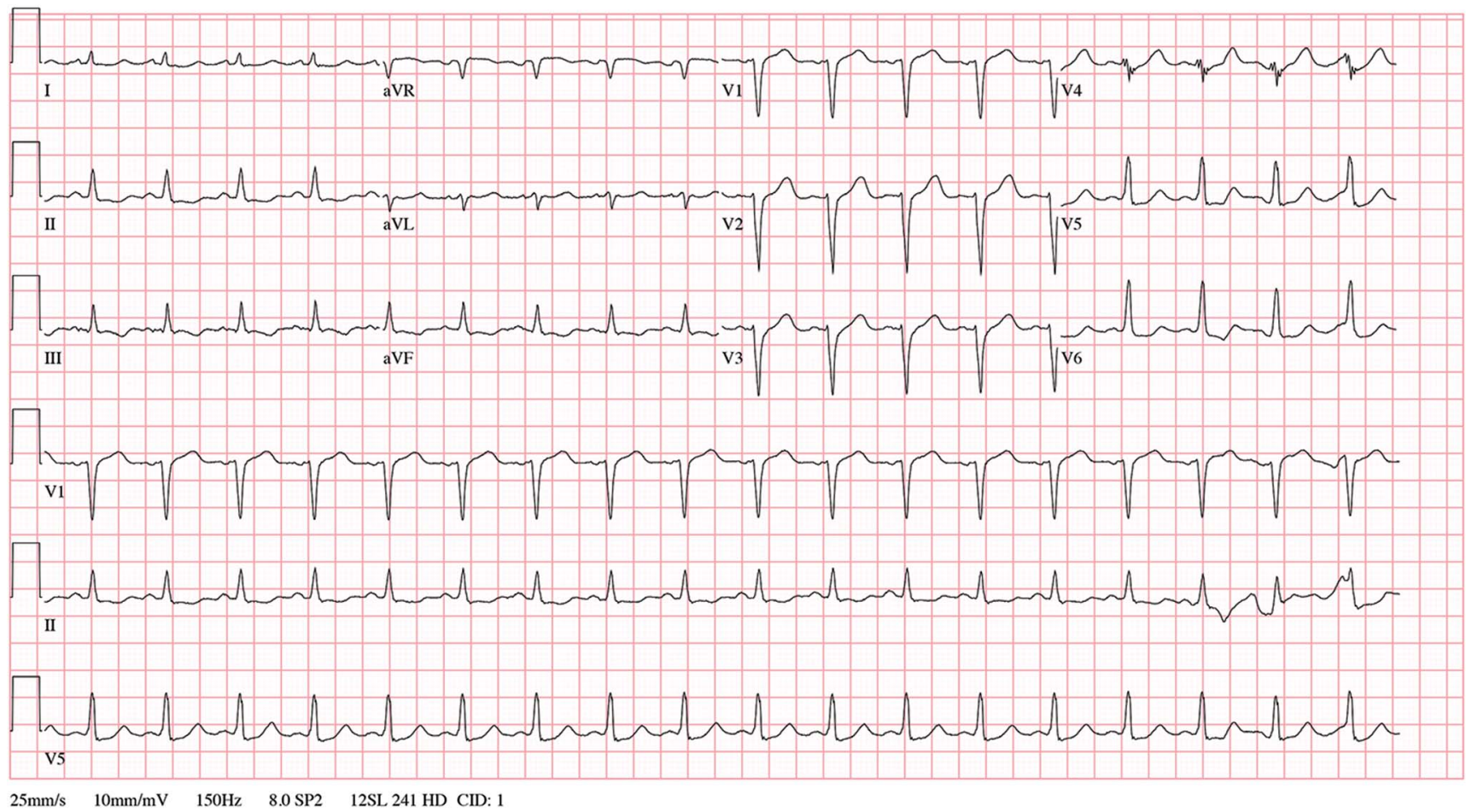

Figure 3. Electrocardiogram before ventricular tachycardia cardiac arrest showing sinus tachycardia. 
acute ventricular unloading has a QT prolonging effect. ${ }^{8,9}$ Our patient had an ECG before recovery from ventricular tachycardia arrest that showed prolonged QTc (Figure 3). However, the patient event coincided with device malpositioning and promptly resolved with Impella device manipulation, so improper positioning was the plausible mechanism. Two possible theories could explain the patient event. First, the presence of both the Impella inlet and outlet outside the ventricular cavity can lead to acute failure of the ventricular offloading. ${ }^{10}$ Consequently, the patient may experience acute ischemia because of the decreased coronary flow and the increased wall tension and oxygen demand. Second, the direct contact between the Impella catheter and the aortic valve can also induce conduction changes that can trigger arrhythmia. ${ }^{11}$ Echocardiogram-guided assessment and correction of the Impella position can be performed at the bedside, and the position correction often solves the arrhythmias induced by the Impella device. ${ }^{12}$ Therefore, careful hemodynamic, laboratory, and imaging assessment of patients with Impella devices and arrythmias should be done at the bedside prior to considering replacement or positioning of the device in the catheterization laboratory.

\section{CONCLUSION}

Patients in cardiogenic shock who require Impella mechanical circulatory support are susceptible to arrhythmias. However, device-induced arrhythmias require careful hemodynamic, laboratory, and imaging assessment at the bedside. Our case highlights the need for immediate bedside echocardiography when device misplacement is suspected, especially during the workup of a new arrhythmia.

\section{ACKNOWLEDGMENTS}

Amir Kaki, MD is a speaker and proctor for ABIOMED. The other authors have no financial or proprietary interest in the subject matter of this article.

\section{REFERENCES}

1. Nalluri N, Patel N, Saouma S, et al. Utilization of the Impella for hemodynamic support during percutaneous intervention and cardiogenic shock: an insight. Expert Rev Med Devices. 2017 Oct;14(10):789-804. doi: 10.1080/17434440.2017.1374849.

2. Burzotta F, Trani C, Doshi SN, et al. Impella ventricular support in clinical practice: collaborative viewpoint from a European expert user group. Int J Cardiol. 2015 Dec 15;201:684-691. doi: 10.1016/j.jijcard.2015.07.065.

3. Basir MB, Schreiber TL, Grines CL, et al. Effect of early initiation of mechanical circulatory support on survival in cardiogenic shock. Am J Cardiol. 2017 Mar 15;119(6):845-851. doi: 10.1016/j.amjcard.2016.11.037.

4. Abaunza M, Kabbani LS, Nypaver T, et al. Incidence and prognosis of vascular complications after percutaneous placement of left ventricular assist device. J Vasc Surg. 2015 Aug;62(2):417-423. doi: 10.1016/j.jvs.2015.03.040.

5. Dangas GD, Kini AS, Sharma SK, et al. Impact of hemodynamic support with Impella 2.5 versus intra-aortic balloon pump on prognostically important clinical outcomes in patients undergoing high-risk percutaneous coronary intervention (from the PROTECT II randomized trial). Am J Cardiol. 2014 Jan 15;113(2):222-228. doi: 10.1016/j.amjcard.2013.09.008.

6. Ouweneel DM, Eriksen E, Sjauw KD, et al. Percutaneous mechanical circulatory support versus intra-aortic balloon pump in cardiogenic shock after acute myocardial infarction. J Am Coll Cardiol. 2017 Jan 24;69(3):278-287. doi: 10.1016/j.jacc.2016.10.022.

7. Peberdy MA, Gluck JA, Ornato JP, et al. American Heart Association Emergency Cardiovascular Care Committee; Council on Cardiopulmonary, Critical Care, Perioperative, and Resuscitation; Council on Cardiovascular Diseases in the Young; Council on Cardiovascular Surgery and Anesthesia; Council on Cardiovascular and Stroke Nursing; and Council on Clinical Cardiology. Cardiopulmonary resuscitation in adults and children with mechanical circulatory support: a scientific statement from the American Heart Association. Circulation. 2017 Jun 13;135(24):e1115-e1134. doi: $10.1161 /$ CIR.0000000000000504.

8. Grzywacz FW, Piacentino V 3rd, Marble J, et al. Effect of acute unloading via head-up tilt on QTc prolongation in patients with ischemic or non-ischemic cardiomyopathy. Am J Cardiol. 2006 Feb 1;97(3):412-415.

9. Harding JD, Piacentino V 3rd, Rothman S, Chambers S, Jessup $M$, Margulies KB. Prolonged repolarization after ventricular assist device support is associated with arrhythmias in humans with congestive heart failure. $J$ Card Fail. 2005 Apr;11(3):227-232.

10. Acou WJ, Bertagnolli L, Hindricks G, Arya A. Positional ventricular tachycardia in left ventricular assist device: a new frontier in ventricular tachycardia ablation. Eur Heart J. 2014 Jan;35(2):65. doi: 10.1093/eurheartj/eht325.

11. Yildirim $Y$, Pecha $S$, Reichenspurner $H$, Deuse T. Mechanically induced ventricular tachycardia by the HeartWare ventricular assist device. ASAIO J. 2014 Jan-Feb;60(1):124-126. doi: 10.1097/MAT.0000000000000019.

12. Catena E, Milazzo F, Merli M, et al. Echocardiographic evaluation of patients receiving a new left ventricular assist device: the Impella recover 100. Eur J Echocardiogr. 2004 Dec;5(6):430-437.

This article meets the Accreditation Council for Graduate Medical Education and the American Board of Medical Specialties Maintenance of Certification competencies for Patient Care and Medical Knowledge. 